\title{
Simulated Low-Support Voltammetry: Deviations from Ohm's Law
}

Christopher Batchelor-McAuley*, Kamonwad Ngamchuea and Richard G. Compton

* corresponding author: Christopher Batchelor-McAuley, Department of Chemistry, Physical \& Theoretical Chemistry Laboratory, Oxford University, South Parks Road, Oxford, OX1 3QZ, United Kingdom

Email: christopher.batchelor-mcauley@chem.ox.ac.uk Tel: +44(0)1865 275448

\begin{abstract}
To what extent can the resistance of a voltammetric system be considered a constant and well described by Ohm's law? Many real electrochemical systems are neither fully in the macro or microscopic limit. Through simulation this work looks at the transition between the steady-state and transient (macroelectrode) mass-transport limits for electrochemical systems, focusing on the influence of ohmic drop on the voltammetric response. Approximate analytical expressions are provided to yield an estimate of the minimum electrolyte to analyte support ratio required to obtain a diffusion only voltammetric response in the absence of convection. Subsequently, this work evidences how for systems in which the electrolyte to analyte ratio is less than unity and the dimensionless scan rate is less than $\sim 400$, then the cell resistance cannot be considered constant. Furthermore, it is demonstrated how under low support conditions the time required to reach voltammetric steady-state is greater than is found for diffusion only systems- this arises due to significant changes in the electrolyte concentrations in the vicinity of the electrode during the course of the voltammetric scan.
\end{abstract}




\section{Introduction}

In a quiescent solution and for an ideal-dilute system, the flux of the jth species $\left(j_{j} / \mathrm{mol} \mathrm{m}^{-2}\right)$ can be described via the Nernst-Planck equation ${ }^{1}$ :

$j_{j}=-D_{j} \nabla c_{j}-z_{j} u_{j} c_{j} \nabla \phi$

where $D_{j}$ is the diffusion coefficient $\left(\mathrm{m}^{2} \mathrm{~s}^{-1}\right), c_{j}$ is the concentration $\left(\mathrm{mol} \mathrm{m}{ }^{-3}\right), z_{j}$ is the charge of the ion, $u_{j}$ is the mobility of the ion $\left(\mathrm{m}^{2} \mathrm{~V}^{-1} \mathrm{~s}^{-1}\right)$, and $\phi$ is the local electrostatic potential (V). In a solution of uniform composition this expression in combination with the assumption of electroneutrality and the conservation of charge leads to both Ohm's law (equation 2) and Laplace's equation for the potential (equation 3); derivations of these expressions are provided in the SI Section 1 and in reference [1].

$\mathrm{i}=-\kappa \nabla \phi$

$\nabla^{2} \phi=0$

where $\kappa\left(\mathrm{C} \mathrm{s}^{-1} \mathrm{~V}^{-1} \mathrm{~m}^{-1}\right)$ is the ionic conductivity of the solution. In his well cited work ${ }^{2}$ Newman used the above two expressions to provide an estimate for the resistance (R/ $/ \Omega$ ) of an ionic current flowing to a disc embedded in a plane, yielding the expression:

$R_{S T, d i s c}=\frac{1}{4 \kappa a}$

where a is the radius of the disc. Rst is the cell's static resistance and corresponds to the systems resistance when the electrochemical cell is not polarised, as may be measured using $\mathrm{AC}$ techniques with small potential amplitudes. As an aside, Equation 4 is formally equivalent to the contact resistance between two metals as given by the Holm-Maxwell expression for a constriction resistance. ${ }^{3}$ In his seminal treatise Clerk Maxwell ${ }^{4}$ attributes the solving of this problem (Laplace's equation for a disc in an infinite plane) to Strutt ${ }^{5}$ who dealt with an analogous system while studying pipe organs. Consequently, these equations have broader significance and applicability outside of the field of electrochemistry. However, for electrolyte solutions expressions 2 and 3 are predicated on the solution's 
ionic composition being uniform. During the course of an electrochemical reaction the concentrations of both electroactive and electro-inactive species are altered in the vicinity of the electrode. Consequently, the question arises as to under what conditions can we justifiably use Ohm's law to describe an electrochemical system?

Since the 1980 s when work was done by, amongst others, ${ }^{6-7}$ Oldham ${ }^{8}$, Amatore, ${ }^{9}$ and Bond $^{10}$ it has been recognised that microelectrodes enable electrochemical reactions to be studied in the absence of excess supporting electrolyte. But for such systems the cell resistance does not necessarily obey Ohm's law. ${ }^{8}$ Theoretical investigations have provided significant insight into their behaviour; however, due to the complexity of the problem both numerical ${ }^{11}$ and analytical ${ }^{8}$ work describing low support electrochemical systems at microelectrode have often been limited to considering systems at true steady-state. Experimentally, much of the original work on studying low support voltammetry was undertaken using organic solvents. This arose, in part, due to the issue of dissolved carbon dioxide forming carbonate ions in aqueous solutions and the challenges of removing this ionic species from the cell. It is only more recently that a method has been developed for studying electrochemical reactions in aqueous media in the near absence of electrolyte. ${ }^{12} \mathrm{~A}$ notable feature of experimental voltammograms measured in very low support conditions (ca. micomolar electrolyte) - even though the microelectrode voltammetric response may reach a steady-state current at high overpotentials - is the propensity for the voltammetric response to show a significant degree of hysteresis; the redox reaction is commonly easier on the backward scan than the forward.

Beyond the micron scale at the nanoscale, it has also been recently experimentally shown to be possible to drive electrochemical reactions in the absence of excess supporting electrolyte. Work by some of the authors has demonstrated that the oxidation of hydrogen can be studied at individual platinum nanoparticles in the absence of additional electrolyte. ${ }^{13}$ Due to the extreme sensitivity of some nanoparticle suspensions to the presence of ionic species the ability to study their electrochemical reactions without the addition of salt is highly beneficial. However, we note as a point of stark contrast, the oxidation of individual silver nanoparticles seemingly requires the presence of millimolar 
concentrations of a halide containing electrolyte. ${ }^{14-15}$ The situation of Ohmic drop for nanoscale systems is complicated by the likely breakdown of the assumption of electroneutrality at these length scales and will not be dealt with in the present work.

This work serves to first provide a concise summary of the relevant research and results to date on resistance in voltammetric systems where electroneutrality is an excellent approximation. This work focuses solely on cases in which the analyte is not charged and undergoes a one-electron transfer process. Although the results are presented for in terms of an oxidation the results are equally applicable to reduction. Furthermore the systems is considered to have no appreciable forced or natural convection. Second, through the use of numerical simulation solving the Nernst-Plank-Poisson equation set with the application of a zero-field approximation at the electrochemical interface, the applicability and limitations in the use of Ohm's law to describe an electrochemical system is probed. This simulation is undertaken to answer the question; for transient (macroelectrode), steady-state (microelectrode) and mixed (transitional) diffusional systems, over the course of a voltammetric experiment to what extent can the system's resistance be considered constant?

\section{Theory}

In this work a numerical model is presented which accounts for the influence of the electrolyte concentration (species $\mathrm{M}^{\mathrm{z}+}$ and $\mathrm{X}^{\mathrm{z}-}$ ) upon the electrochemical reaction via solving the Nernst-Plank-Poisson equations in the solution phase. The numerical simulation provided in this work closely mirrors that outlined in the literature; ${ }^{16-17}$ consequently, the methods used will only be briefly summarised here. The model is limited to considering a neutral solution phase species, A, undergoing a one electron oxidation to form $\mathrm{B}^{+}$, as given by:

$$
A_{(a q)} \rightleftharpoons B_{(a q)}^{+}+e^{-}
$$

The used dimensionless parameters are tabulated in Table 1. 
Table 1: Definitions of the Dimensionless Parameters used in this Work

\begin{tabular}{|c|c|c|}
\hline $\begin{array}{l}\text { SI unit } \\
\text { parameters }\end{array}$ & Interpretation & $\begin{array}{l}\text { Dimensionless } \\
\text { parameters }\end{array}$ \\
\hline $\mathrm{a}(\mathrm{m})$ & $\begin{array}{l}\text { Radius of the } \\
\text { electrode }\end{array}$ & \\
\hline $\mathrm{r}(\mathrm{m})$ & Space coordinate & $\mathrm{R}=\mathrm{r} / \mathrm{a}$ \\
\hline $\mathrm{c}(\mathrm{mM})$ & Concentration & $\mathrm{C}_{\mathrm{j}}=\mathrm{C}_{\mathrm{j}} / \mathrm{c}_{\mathrm{A}, \mathrm{bulk}}$ \\
\hline$D\left(m^{2} s^{-1}\right)$ & Diffusion Coefficient & $d_{j}=D_{j} / D_{A}$ \\
\hline$t(s)$ & Experimental time & $\mathrm{T}=\mathrm{D}_{\mathrm{A}} \mathrm{t} / \mathrm{a}^{2}$ \\
\hline $\mathrm{k}_{0}\left(\mathrm{~m} \mathrm{~s}^{-1}\right)$ & $\begin{array}{l}\text { Rate of electron } \\
\text { transfer }\end{array}$ & $\mathrm{K}_{0}=\mathrm{k}_{0} \mathrm{a} / \mathrm{D}_{\mathrm{A}}$ \\
\hline$\phi(V)$ & $\begin{array}{l}\text { Electrostatic } \\
\text { Potential }\end{array}$ & $\Phi=\phi \mathrm{F} / \mathrm{RT}$ \\
\hline$E(V)$ & Electrode Potential & $\Theta=\left(E-E_{f}\right) F / R T$ \\
\hline$v\left(\mathrm{~V} \mathrm{~s}^{-1}\right)$ & Scan Rate & $\sigma=a^{2} F v / D_{A} R T$ \\
\hline
\end{tabular}




\section{Nernst Plank Poisson (NPP) Equations}

Under spherical coordinates and using dimensionless parameters the Nernst-Planck equation is: ${ }^{17}$

$\frac{\partial C_{j}}{\partial T}=d_{j}\left(\frac{\partial^{2} C_{j}}{\partial R^{2}}+\frac{2}{R} \frac{\partial C_{j}}{\partial R}\right)+d_{j} Z_{j}\left(\frac{\partial C_{j}}{\partial R} \frac{\partial \Phi}{\partial R}+C_{j} \frac{\partial^{2} \Phi}{\partial R^{2}}+C_{j} \frac{2}{R} \frac{\partial \Phi}{\partial R}\right)$

The Poisson equation is used to relate the dimensionless potential to the local electric charge density, again in dimensionless form the equation is:

$-R_{e}^{2} \sum_{j} z_{j} C_{j}=\frac{\partial^{2} \Phi}{\partial R^{2}}+\frac{2}{R} \frac{\partial \Phi}{\partial R}$

where $R_{e}$ represents the scale of the electrode compared to the Debye length.

$R_{e}=\sqrt{\frac{F^{2} c_{A} a^{2}}{R T \epsilon_{s} \epsilon_{0}}}$

$\epsilon_{0}$ is the permittivity of free space $\left(8.854 \times 10^{-12} \mathrm{~F} \mathrm{~m}^{-1}\right)$ and $\epsilon_{s}$ is the relative permittivity with a value of 80.1 for the case of water. Although the above model does not a priori assume uniform electroneutrality in reality at the length scales being studied in this work (i.e. micron sized electrodes and above) electroneutrality is a good approximation.

\section{Boundary Conditions}

The initial $(T=0, R \geq 1)$ and outer boundary conditions $\left(T>0, R=R_{\max }\right)$ are $C_{A}=1, C_{B}=0, \Phi=$

$0(\mathrm{~T}=0, \mathrm{R} \geq 1), \mathrm{C}_{\mathrm{M}}=\mathrm{C}_{\mathrm{X}}=\mathrm{C}^{*}$ sup, $\left.\Phi\left(\mathrm{R}=\mathrm{R}_{\max }\right)+\mathrm{R}_{\max } \frac{\partial \Phi}{\partial R}\right)_{R=R_{\max }}=0\left(\mathrm{~T}>0, \mathrm{R}=\mathrm{R}_{\max }\right)$. At the electrode interface the zero-field approximation ${ }^{18}$ is used as a boundary condition implying complete decoupling of the double layer from the potential distribution near the electrode. Moreover, the flux of the electrolyte cation and anion are similarly set to zero at the electrode interface as expressed below. 
$\left(\frac{\partial \Phi}{\partial R}\right)_{R=1}=\left(\frac{\partial \mathrm{C}_{M}}{\partial R}\right)_{R=1}=\left(\frac{\partial \mathrm{C}_{X}}{\partial R}\right)_{R=1}=0$

The Butler-Volmer formalism is used to describe the electron transfer kinetics, the overpotential is modified to account for the drop in potential across the solution phase.

$\left.\frac{\partial C_{A}}{\partial R}\right)_{R=1}=K_{0} \exp \left(\beta\left(\Theta-\Phi_{0}\right)\right) C_{A}-K_{0} \exp \left((\beta-1)\left(\Theta-\Phi_{0}\right)\right) C_{B}$

here $\beta$ is the transfer coefficient, $K_{0}$ is the dimensionless standard rate constant, $\Theta$ is the applied dimensionless potential and $\Phi_{0}$ is the dimensionless solution phase potential adjacent to the electrochemical interface. Additionally from the conservation of mass:

$\left.\left.d_{B} \frac{\partial C_{B}}{\partial R}\right)_{R=1}=-\frac{\partial C_{A}}{\partial R}\right)_{R=1}$

The resulting Jacobian matrix has 15 diagonals when the unknowns are ordered $\mathrm{C}_{\mathrm{A}, 0}, \mathrm{C}_{\mathrm{B}, 0 \text {, }}$ $\Phi_{0}, \mathrm{C}_{\mathrm{M}, 0}, \mathrm{CX}_{\mathrm{X}, 0}, \mathrm{C}_{\mathrm{A}, 1}, \mathrm{C}_{\mathrm{B}, 1}, \Phi_{1}, \mathrm{C}_{\mathrm{M}, 1}, \mathrm{CX}_{\mathrm{X}, 1}, \ldots$

\section{Numerical Methods}

The NPP set of equations were solved using a finite difference method. Between the electrode surface and a maximum distance away from the electrode $\left(R_{\max }=1+\right.$ $6 \sqrt{d_{\max } T_{\max }}$ ) an expanding grid is used to discretise the space, this grid provides dense gridding near the electrode surface below a threshold value of $R_{s}+1$ and expands at rate proportional to $\gamma_{R}$ beyond $\mathrm{R}_{\mathrm{s}}+1$ as given by;

$R[i]=R[i-1]+\gamma_{R} R_{S} \quad 1<\mathrm{R}<\mathrm{R}_{\mathrm{S}}+1$

$R[i]=R[i-1]+\gamma_{R}(R[i-1]-1) \quad \mathrm{R}_{\max }>\mathrm{R}>\mathrm{R}_{\mathrm{s}}+1$

here $i$ relates to the grid indexing. Optimal grid parameters were found to be $\gamma_{R}=$ $5 \times 10^{-3}$ and $R_{s}=2 \times 10^{-3}$. The potential of the system was varied linearly with time, a potential step of $2 \times 10^{-4} \mathrm{~V}$ between each subsequent simulated time step was used throughout this work. 
Equations 6 and 7 were discretised using a central difference scheme which for a general function $\mathrm{F}$ is given as;

$\frac{\partial F}{\partial R}=\frac{F_{i+1}-F_{i}}{2 \Delta R_{+}}+\frac{F_{i}-F_{i-1}}{2 \Delta R_{-}}$

$\frac{\partial^{2} F}{\partial R^{2}}=\frac{2\left(F_{i+1}-F_{i}\right)}{\Delta R_{+}\left(\Delta R_{+}+\Delta R_{-}\right)}+\frac{2\left(F_{i}-F_{i-1}\right)}{\Delta R_{-}\left(\Delta R_{+}+\Delta R_{-}\right)}$

where $\Delta R_{+}$and $\Delta R_{-}$are the forward and backward differences in the grid spacing respectively.

The discretised set of simultaneous nonlinear equations was solved iteratively via the Newton-Raphson method, as a convergence tolerance criterion the mean absolute change in the concentrations must be less than $1 \times 10^{-8}$. All simulations were scripted in Python 3.5, CPU intensive routines were precompiled into $\mathrm{C}$ using Cython. The diagonally banded matrix system resulting from the Newton-Raphson method was solved using the LAPACK 'gbsv' routine as provided as part of the SciPy package.

\section{Results and Discussion}

The occurrence of a current (Faradaic or capacitative) at an electrochemical interface leads to a build-up of charge in the vicinity of the interface. In the absence of excess supporting electrolyte this charge separation leads to an appreciable electric field that extends away from the surface. This electric field causes two significant effects; first ions move under the influence of the field (migration) altering the systems mass-transport and second the electrochemical reaction is, at least partially, inhibited due to the decrease in the potential experienced at the electrochemical interface (Ohmic drop). This drop in potential across the solution phase is observed to distort any measured voltammogram. In the case of a reversible process at a macroelectrode this can lead to a significant increase in the voltammetric peak-to-peak separation. Such distortions may be misinterpreted as relating to the electron transfer kinetics being slow. The larger the resistance of a cell the more distorted the voltammetry is. 
In a three electrode system where the counter electrode is significantly larger than the working electrode this drop in potential occurs predominantly in the vicinity of the working electrode; outside of the double layer the potential drops over a length scale comparable to that of the electrode radius. It is beneficial to consider this problem in analogy with a constriction resistance where the potential drops across the point of constriction. $^{3}$ For a hemispherical working electrode the resistance to the flow of an ionic current to the surface, analogous to that of a disk as given in equation 4 , is

$R_{S T, h e m i}=\frac{1}{2 \pi \kappa a}$

Equations 4 and 16 have been previously referred to as the static electrochemical cell resistance. They are approximate estimates of the resistance of the cell and will only be applicable under certain limits. The simulation model provided in this work allows the electrochemical system's resistance at any given time to be readily determined by measuring the simulated potential at the electrode surface $\left(\phi_{0}\right)$ relative to that of the potential of the bulk solution $\left(\phi_{b u l k}\right)$ and dividing by the total electrochemical current, as given by the following expression:

$R_{\text {cell }}=\frac{\phi_{0}-\phi_{\text {bulk }}}{I}$

Using equation 17 to provide a measure of the simulated cell resistance allows us to question under what conditions equation 16 provides an accurate description of the system.

The following three sections summarise the behaviour of an electrochemical cell when using a) a macroelectrode b) a microelectrode and c) the transition between these two regimes. This final section allows us to answer the question, 'under what conditions can an electroanalytical system be viewed as obeying Ohm's law'? 


\section{Transient Limit: Voltammetry at a Macroelectrode}

For a macroelectrode the diffusion layer thickness (the region over which the electroactive species are depleted) is small as compared to the size of the electrode. It has been previously noted through simulation, and confirmed by comparison of the model with experiment, that in this linear diffusion limit the resistance during the course of the voltammetric scan is relatively constant. ${ }^{17}$

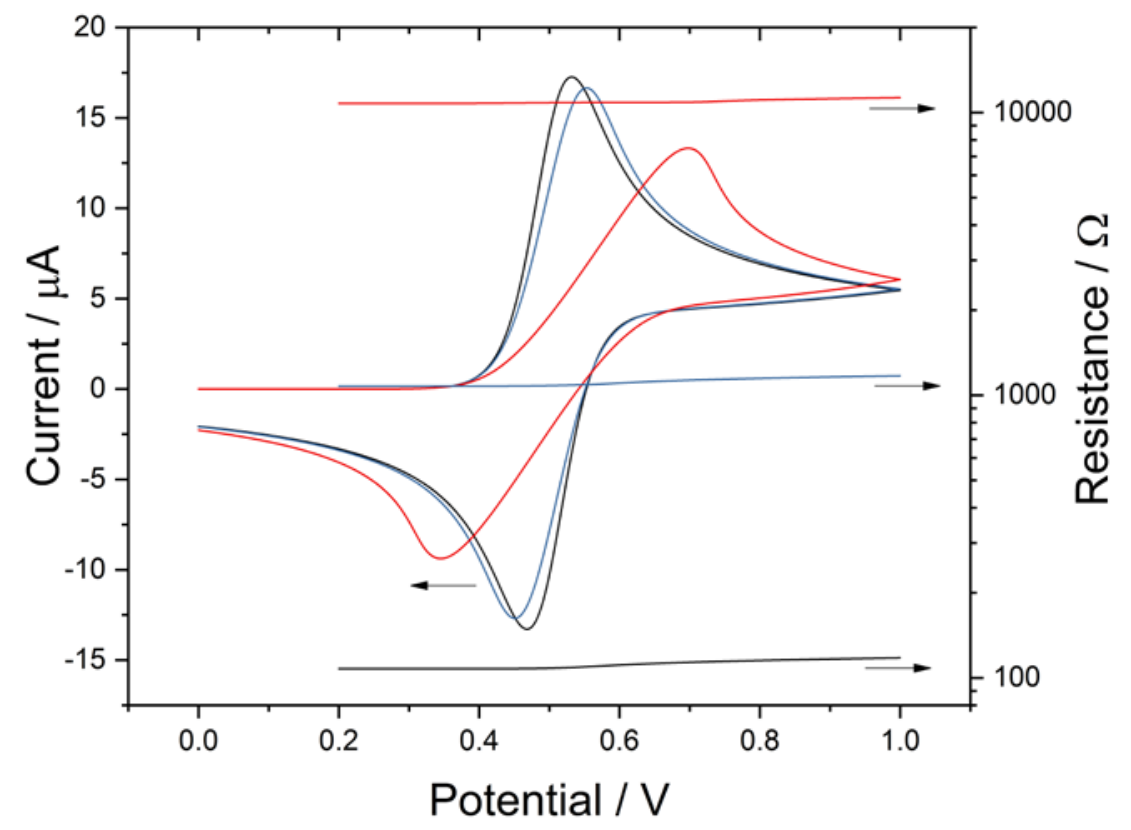

Figure 1: Simulated voltammetric response of a $1 \mathrm{~mm}$ radius hemispherical electrode scanned at $100 \mathrm{mV} \mathrm{s}-1$, for the oxidation of a $1 \mathrm{mM}$ solution of a redox active species $\left(D_{A}=1 \times 10^{-9} \mathrm{~m}^{2} \mathrm{~s}^{-1}\right)$, where the supporting electrolyte $\left(D_{\text {sup }}=2 \times 10^{-9} \mathrm{~m}^{2} \mathrm{~s}^{-1}\right)$ concentration is, black; $100 \mathrm{mM}$, blue; $10 \mathrm{mM}$ and red; $1 \mathrm{mM}$. Also plotted is the simulated resistance of the system as measured on the forward scan, the different colours correspond to the associated voltammogram as simulated with differing electrolyte concentrations. The resistance is measured using the definition given in equation 17 and is the potential drop across the solution phase divided by the voltammetric current. The electron transfer rate has been set arbitrarily high at $10 \mathrm{~m} \mathrm{~s}^{-1}$, to ensure the redox processes are fully electrochemically reversible, $\mathrm{E}_{\mathrm{f}}=0.5 \mathrm{~V}$.

Figure 1 presents the simulated voltammetric response for the reversible oxidation of a 1 $\mathrm{mM}$ solution of an electroactive analyte at a $1 \mathrm{~mm}$ radius hemispherical electrode at a scan rate of $100 \mathrm{mV} \mathrm{s}^{-1}$, in the presence of 100,10 and $1 \mathrm{mM}$ of a monovalent electrolyte. Overlaid on this is the simulated resistance ( $\left.\mathrm{R}_{\text {cell }}\right)$ of the system during the course of the scan as determined through the use of equation 17. Through the use of the Nernst-Einstein equation (see SI section 2) the molar conductivity of the solution can be determined to be 
$15.0 \mathrm{mS} \mathrm{m} \mathrm{mol}^{-1}$ (Dsup $=2 \times 10^{-9} \mathrm{~m}^{2} \mathrm{~s}^{-1}$ ); consequently, for a hemispherical electrode, in combination with equation 16 the system static resistance is predicted to be $106 \Omega, 1.06$ $\mathrm{k} \Omega$ and $10.6 \mathrm{k} \Omega$ for the electrolyte concentrations 100,10 and $1 \mathrm{mM}$ respectively. As can be seen by comparison of these values to the resistances presented in Figure 1, the simulated and analytically calculated static cell resistance differ by no more than $7 \%$ of the course of the forward scan. Although the systems resistance is not strictly Ohmic, such small discrepancies are likely not easily physically measurable. Moreover, the largest deviations occur at high overpotential when the voltammetric response is under diffusional control and hence measurement of the solution phase potential drop is not easily achieved under such rate determining conditions. Consequently, in the case of macroelectrode voltammetry, experimental results can likely be justifiably corrected to account for the influence of ohmic drop by utilising simpler analytical correction methods. ${ }^{19}$ As a slight caveat this result does not necessarily hold in the case of rotating disc electrodes where the system is under forced convections and some experimental results indicate the potential inapplicability of equation 4 to such systems. ${ }^{20}$

In this limit of a macroscopic electrode the use of equation 16 to predict the resistance of the cell is accurate due to the differing lengths scales involved; the potential drop, induced by the occurrence of the electrochemical reaction, varies over a distance comparable to the size of the electrode ${ }^{21}$ (ca. $1 \mathrm{~mm}$ ) whereas the occurrence of the electrochemical reaction only alters the concentration of the species within the diffusion layer $(\delta \sim \sqrt{2 D t}$ ca. tens of microns). This accuracy of the static resistance in defining the resistance of the cell allows us to provide an approximate expression defining the minimum electrolyte concentration needed to ensure a macroelectrode voltammogram is not significantly distorted due to ohmic drop effects. The expressions provided below (equation 18 for a hemispherical electrode and equation 19 for a planar electrode) assume a reversible one-electron process at a macroelectrode. The ionic conductivity is estimated via the Nernst-Einstein equation and assumes the presence of a monovalent electrolyte where the diffusion coefficients of the electrolyte anion and cation are equal. We make the further approximation that the voltammetric peak current is assumed to be unaltered by Ohmic distortion (only valid for 
small Ohmic drops). Further details of the derivation of equations 18 and 19 and validation via simulation can be found in the SI section 3 .

Hemispherical electrode $\quad \frac{C_{\text {sup }}}{C_{A}}=0.223 \sqrt{\frac{R T}{F}} \frac{D_{A}^{0.5} v_{\text {sup }}^{0.5}\left|V_{\text {drop }}\right|}{D_{\text {a }} \mid}$

Planar electrode $\quad \frac{C_{\text {sup }}}{C_{A}}=0.0558 \pi \sqrt{\frac{R T}{F}} \frac{D_{A}^{0.5} v^{0.5} a}{D_{\text {sup }}\left|V_{\text {drop }}\right|}$

$\mathrm{C}_{\text {sup }}$ and $\mathrm{c}_{\mathrm{A}}$ are the solution phase concentrations of the supporting electrolyte and electroactive analyte respectively, $v$ is the voltammetric scan rate $\left(\mathrm{V} \mathrm{s}^{-1}\right)$ and all other symbols are as previously used. The above expression uses the magnitude of the solution phase potential drop ( $V_{\text {drop }}$ ) to estimate the needed supporting electrolyte concentration. In a voltammogram $V_{\text {drop }}$ can be approximately viewed as the potential shift in the forward peak current from its expected position if the electrode reaction was fully supported. For example, for a potential drop ( $\left.\mathrm{V}_{\mathrm{drop}}\right)$, and hence error in the position of the voltammetric forward peak current of no more than $3 \mathrm{mV}$ at a hemisphere of radius $1 \mathrm{~mm}$ (at a scan rate of $100 \mathrm{mVs}^{-1}$ and taking all diffusion coefficients to have a value of $1 \times 10^{-9} \mathrm{~m}^{2} \mathrm{~s}^{-1}$ ) then the above expression predicts a required electrolyte support ratio $\left(c_{\text {sup }} / c_{A}\right)$ of 119 . For a macrodisc of equivalent radius the required support ratio is predicted to be 94 . In the case where the diffusion coefficient of the analyte is four times less than that of the electrolyte $\left(D_{A}=5 \times 10^{-10} \mathrm{~m}^{2} \mathrm{~s}^{-1}\right.$ and $\left.D_{\text {sup }}=2 \times 10^{-9} \mathrm{~m}^{2} \mathrm{~s}^{-1}\right)$ this value for the required electrolyte support ratio decreases to 42 (33 for a planar macroelectrode). As outlined in the SI section 3, equations 18 and 19 are also approximately applicable to the situation in which the electroactive reactant is charged.

\section{Steady-State Limit: Voltammetry at a Microelectrode}

The following summarises the voltammetric behaviour of a microelectrode under true steady-state conditions at low support concentrations. Before proceeding a few important points should be made about this limiting case. First, under true steady-state conditions the problem of solving the steady-state diffusion current and the problem of determining the 
cell conductance are congruent. ${ }^{22}$ To restate this point, the concentration profile and potential profile around the electrode are of the same length scale and geometry; consequently, under many conditions the Ohmic drop to a microelectrode is found to be independent of both its size and shape. To exemplify this further, consider a microdisc electrode, the expected diffusional current is proportional to the electrode radius. Conversely, in the high support limit the solution phase resistance is proportional to the inverse of the electrode radius; hence, at steady-state the expected potential drop ( $V=I R)$ can be seen to be independent of the dimension of the electrode. This result also holds true under low support conditions as reflected later in equation 23. Second, under steady-state conditions the ionic current is transported solely by the electroactive species and not the electrolyte..$^{23}$ Third, the concentration of the electrolyte in the vicinity of the electrode can be significantly altered during the course of an electrochemical reaction at a microelectrode. ${ }^{7}$ This change in the local ionic strength leads to an alteration of the cells resistance during the course of voltammetric scan. Hence the term 'Ohmic' drop can be somewhat misleading in the case of microelectrode voltammetry in that it wrongly implies the cell resistance to be constant.

For the electrochemical reaction presented in equation 5 then the steady-state resistance $\left(R_{s s}\right)$ of an electrochemical cell containing a microelectrode is in the case where the reactant is not charged, at steady-state: 8

$R_{S S}=\frac{R T}{I F} \ln \left[1+\frac{I}{4 \pi F a D_{B} C_{\text {sup }}}\right]$

where $\mathrm{D}_{\mathrm{B}}$ is the diffusion coefficient of the product $\left(\mathrm{B}^{+}\right)$of the electrochemical reaction. Equation 20 applies at all currents and not just when the electrochemical reaction is diffusionally limited. At low potentials, as I $\rightarrow 0$, the steady-state resistance becomes

$R_{S S, I \rightarrow 0}=\frac{R T}{4 \pi F^{2} a D_{B} C_{S u p}}$ 
Consequently, in the low current limit the steady-state resistance to a microdisc electrode differs from the cells static resistance (as given by equation 16) when the diffusion coefficients of the electrolyte and electroactive product differ:

$R_{S S, I \rightarrow 0}=\frac{D_{\text {sup }}}{D_{B}} R_{S T, h e m i}$

Here we have assumed the diffusion coefficient of the electrolyte anion and cation are equal and expressed above as $\mathrm{D}_{\text {sup. }}$

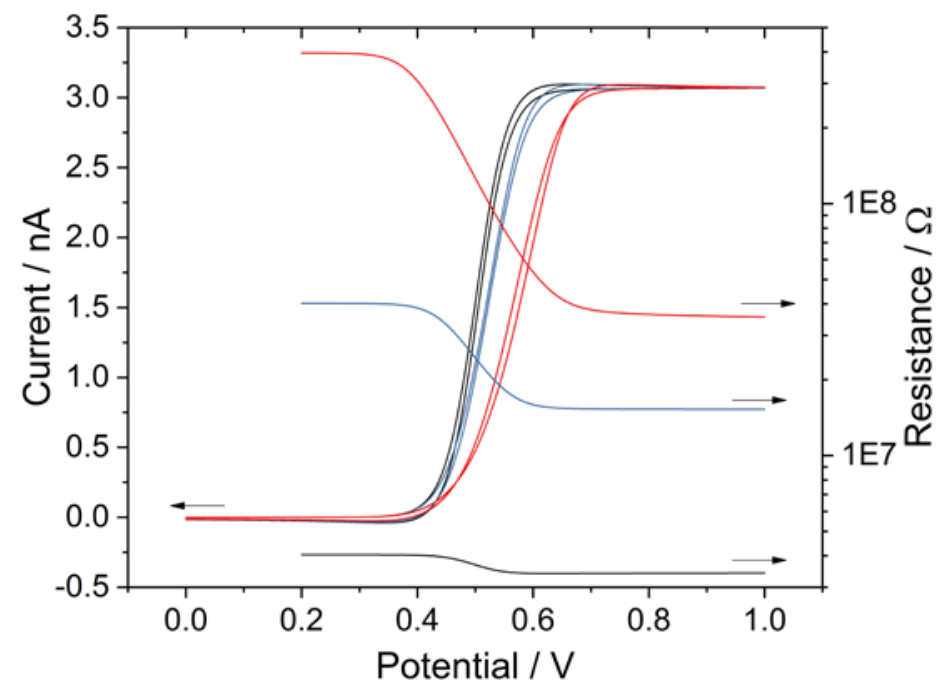

Figure 2: Simulated voltammetric response of a hemispherical $5 \mu \mathrm{m}$ radius electrode scanned at $10 \mathrm{mV} \mathrm{s}{ }^{-1}$, for the oxidation of a $1 \mathrm{mM}$ solution of a redox active species $\left(D_{A}=1 \times 10^{-9} \mathrm{~m}^{2} \mathrm{~s}^{-1}\right)$, where the supporting electrolyte $\left(D_{\text {sup }}\right.$ $=2 \times 10^{-9} \mathrm{~m}^{2} \mathrm{~s}^{-1}$ )concentration is, black; $1.0 \mathrm{mM}$, blue; $0.1 \mathrm{mM}$ and red; $0.01 \mathrm{mM}$. Also plotted is the simulated resistance of the system as measured on the forward scan, the line colours correspond to the differing electrolyte concentrations. The electron transfer rate has been set arbitrarily high at $10 \mathrm{~m} \mathrm{~s} \mathrm{~s}^{-1}$, to ensure the redox processes are fully electrochemically reversible, $\mathrm{E}_{\mathrm{f}}=0.5 \mathrm{~V}$.

Figure 2 presents the simulated voltammetric response for the reversible oxidation of a 1 $\mathrm{mM}$ solution of an electroactive analyte at a $5 \mu \mathrm{m}$ radius hemispherical electrode in the presence of 1.0, 0.1 and $0.01 \mathrm{mM}$ of a monovalent electrolyte at a scan rate of $10 \mathrm{mV} \mathrm{s}^{-1}$. Overlaid on this is the resistance of the system during the course of the scan as determined from equation 17. From equation 21 the predicted low current limit for the resistance are $4.2 \times 10^{6}, 4.2 \times 10^{7}$ and $4.2 \times 10^{8} \Omega$ for electrolyte concentrations of $1.0,0.1$ and $0.01 \mathrm{mM}$ respectively. At low potentials (below ca. $+0.4 \mathrm{~V}$ ), these values are in good agreement with 
those found from simulation and as can be seen in Figure 2. Moreover, during the course of the voltammetric scan the cell resistance decreases as the current increases reaching a second limit at high overpotentials. This decrease occurs due to migration and rearrangement of the ionic species in the electric field adjacent to the electrode, hence leading to an increase in the ionic strength of the solution on the vicinity of the electrode. By substituting in the known expression for the diffusion limited current to a micro hemispherical electrode equation 20, can be used to predicted the expected cell resistance at high overpotentials, where for the simulation conditions used in Figure 2 the predicted cell resistances are found to be $3.4 \times 10^{6}, 15.1 \times 10^{6}$ and $33.3 \times 10^{6}$, this is again in excellent agreement with that found by numerical simulation. Note in the low potential limit the resistance scales linearly with the supporting electrolyte concentration this is not the case in the high potential limit. The ratio of the resistance in the low and high overpotential limits will be examined further in the next section and is quantified via equation 24 . As a further point it is highlighted that these variations in cell resistance are likely less applicable to measurements made using impedance spectroscopy where high frequencies are employed and hence the system is not studied under steady state.

Equation 20 provides a facile route by which the minimum required concentration of supporting electrolyte can be defined for a microelectrode system. Using the half-wave potential as a measure of the voltammetric distortion due to ohmic drop then: ${ }^{24}$

$\frac{c_{\text {sup }}}{c_{A}}=\frac{D_{A}}{4 D_{B}\left(\exp \left(\frac{F V_{d r o p}}{R T}\right)-1\right)}$

Hence, the required support ratio is independent of the size of the electrode and at steadystate does not depend on the diffusion coefficient of the electrolyte. Equation 23 predicts, at steady-state when $\mathrm{D}_{\mathrm{A}}=\mathrm{D}_{\mathrm{B}}$, that for less than a $3 \mathrm{mV}$ drop in potential ( $\mathrm{V}_{\text {drop}}$ ) across the electrochemical cell the required support ratio is 2 . SI section 4 evidences the validity of this expression through comparison with numerical simulation. This SI section also briefly considers the effect of the charge of the reactant, the conclusions of which are: First, the use of a charged reactant tends to minimise the solution phase ohmic drop by increasing the 
solution phase conductivity, equation 20 is not applicable in this situation. The required support ratio for a given potential drop will in the case of a charged reagent be overestimated by the direct application of equation 23. Second, the mass-transport of the reactant is altered by the presence of the electric field leading to the steady-state current being altered. This leads to a situation in which one can ask 'what is the required supporting electrolyte concentration required to ensure the steady-state current is not significantly altered from the diffusion only case'? Crudely, we can still use equation 23 to answer this question; in the absence of a significant potential drop in the solution phase the mass-transport of the charged species is not expected to be significantly influenced by migration. More precisely, for a one-electron oxidation of a negatively charged species the predicted (on the basis of simulations presented in the SI section 4) steady-state current is expected to be with $10 \%$ of that of the diffusion only case when a support ratio of 2 is used. Supporting electrolyte ratios of 10 and 26 , lead to changes of $3 \%$ and $1 \%$ in the magnitude of the expected steady-state current respectively. Precise values for systems with different reactant charges will differ in their exact values but will be similar in magnitude.

\section{Mixed Diffusional Regimes: Microelectrodes not at Steady-State}

In the above it has been highlighted how for a macroelectrode an electrochemical cell can be reasonably viewed as following Ohm's law where the cell resistance is essentially constant and is accurately estimated by the cell static resistance. Conversely in the steadystate limit for a microelectrode due to migration of ions in the vicinity of the electrode altering the ionic strength local to the interface, the cell resistance varies during the course of a voltammetric scan. Many electrochemical systems will lie between these two limits. Consequently, this section seeks to answer two questions; first under what conditions can the electrochemical cell be well described using Ohm's law and second for low support conditions on what voltammetric time scale is steady-state behaviour attained?

To facilitate comparison between the micro and macro limits, the following simulations will assume that all of the ionic species diffusion coefficients are equal; consequently, in accordance with equation 22, in the low current limit both the static and steady-state cell 
resistances will have the same value. In order to assess the magnitude of the change in the cell's resistance during the course of the voltammetric scan the calculated resistance at an overpotential of $0.5 \mathrm{~V}$ is compared to that predicted on the basis of equation 21 and equivalently 16.

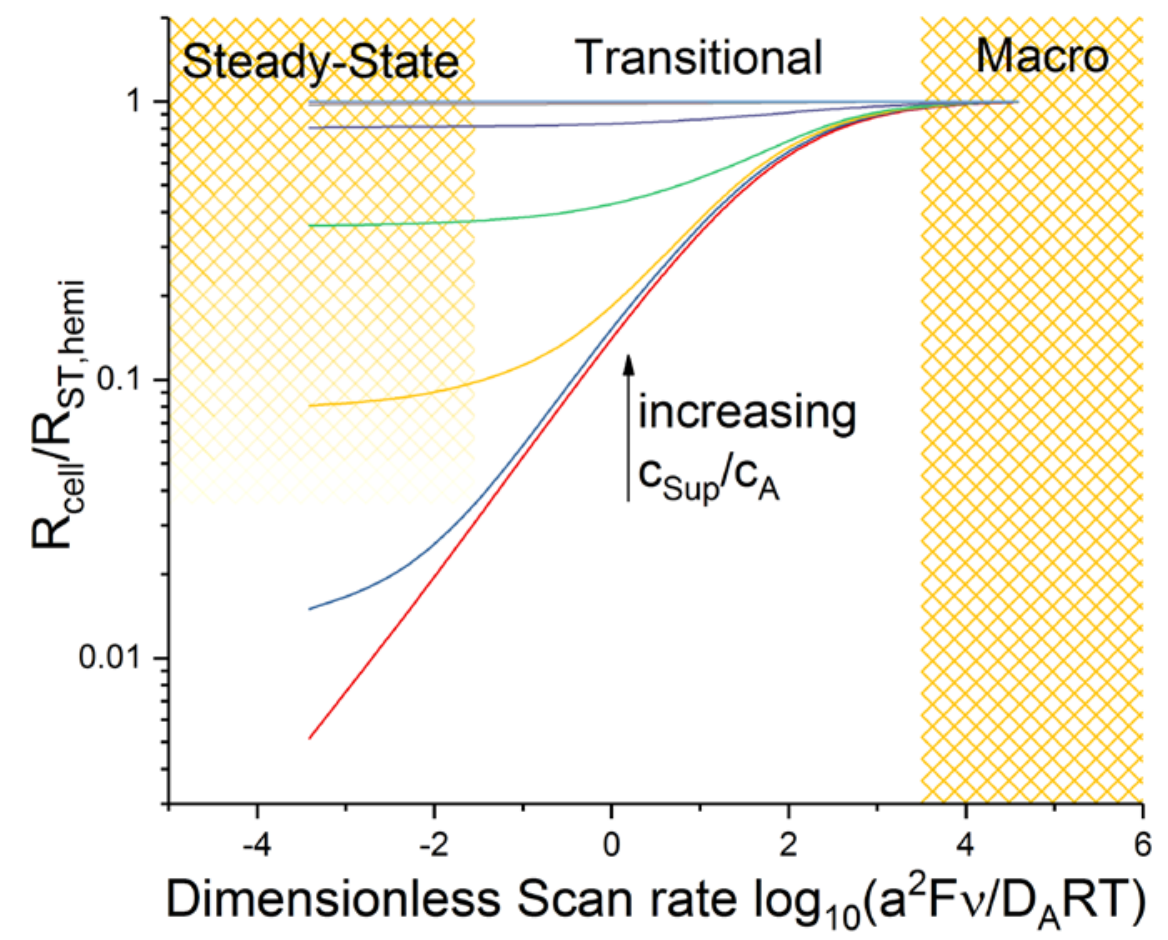

Figure 3: Change in the cell resistance for a system containing a hemispherical electrode, as predicted by simulation and presented as a function of the dimensionless scan rate. The diffusion only limits in which the geometry of the system can either be viewed as being macroscopic (linear diffusion regime) or at steady-state (convergent diffusion regime) are indicated on the Figure, note at low support ratios the system does not reach steady-state even at low scan rates, this is graphically indicated. Resistance change is quantified by measurement of the cell's resistance in accordance with equation 17 , and determined at $0.5 \mathrm{~V}$ past the formal potential for the redox couple, measured resistance is normalised against the cells resistance at low current as determined by equations 12 (macro electrode) and 17 (micro electrode) in the present case where all diffusion coefficients are equal equations 16 and 21 lead to the same result as indicated by equation 22 . Results are presented as a function of the electrolyte support ratio: red, $1 \times 10^{-4}$; blue, $1 \times 10^{-3}$; yellow, $1 \times 10^{-2}$; green, $1 \times 10^{-1}$; purple, $1 \times 10^{0}$; grey, $1 \times 10^{1}$ above this support ratio the cell resistance is essentially invariant during the course of the scan.

Figure 3 depicts the change in the cell resistance during the course of a voltammetric scan and is plotted as a function of the dimensionless scan rate and the support ratio in the range of $1 \times 10^{-4}-1 \times 10^{3}$. In the macroelectrode limit (dimensionless scan rate $\sigma>2920$, as highlighted on Figure 3) for all support ratios the cells resistance does not alter significantly over the course of the scan and $\frac{R_{\text {cell }}}{R_{S T, \text { hemi }}} \rightarrow 1$. Note that here a value of unity 
does not imply that the voltammogram is not distorted due to ohmic drop, this value merely indicates that the cell resistance is constant during the course of the scan. Conversely, in the microelectrode steady-state limit (small $\sigma$ ) and for low support ratios the cell's resistance changes considerably over the course of the scan. For example, at a support ratio of $1 \times 10^{-3}$ and a dimensionless scan rate of 0.001 (equivalent to an electrode with a radius of approximately $1.6 \mu \mathrm{m}$ at a scan rate of $10 \mathrm{mV} \mathrm{s}^{-1}$ with an analyte diffusion coefficient of $1 \times 10^{-9} \mathrm{~m}^{2} \mathrm{~s}^{-1}$ ) over the course of the voltammetric scan the cells resistance decrease by almost two orders of magnitude. In the true steady-state limit the drop in the cell resistance over the course of the scan can be predicted by the ratio of equations 20 and 21 as given by:

$\frac{R_{\text {cell }}}{R_{\text {sS }}}=\frac{2 D_{B} c_{\text {sup }}}{D_{A} c_{A}} \ln \left(1+\frac{D_{A} c_{A}}{2 D_{B} c_{\text {sup }}}\right)$

Here $R_{s s}$ is equal to $R_{S T, h e m i}$ due to the electrolyte and electrode product having equal diffusion coefficients. Hence for the above example in the steady-state limit the drop in the cell resistance will tend towards a ratio of $1.2 \times 10^{-2}$. Furthermore, in agreement with equation 24 , in the low dimensionless scan rate limit the simulated data as presented in Figure 3 shows that the drop in the cells resistance becomes independent of the dimensionless scan rate (and hence independent of the electrode size) in the steady-state regime. It is of distinct interest that the scan rate at which the steady-state regime is reached is sensitive to the support ratio. For a diffusion only system the diffusional steadystate limit is attained at a hemispherical electrode when the dimensionless scan rate has a value of less than $2.1 \times 10^{-2}$ (as determined by the expected current being no more the $3 \%$ greater than that predicted for a true steady-state process), this diffusional threshold is marked on Figure 3. However, at low support ratios the timescale and hence dimensionless scan rate required for the system to attain steady-state (as in the present case indicated by the drop in resistance becoming independent of the dimensionless scan rate) differs from the diffusion only case. Similar results have been previously noted for chronoamperometric ${ }^{25}$ experiments. The following section will exemplify how the differing timescales for attainment of a steady-state behaviour influence the voltammetric response. 

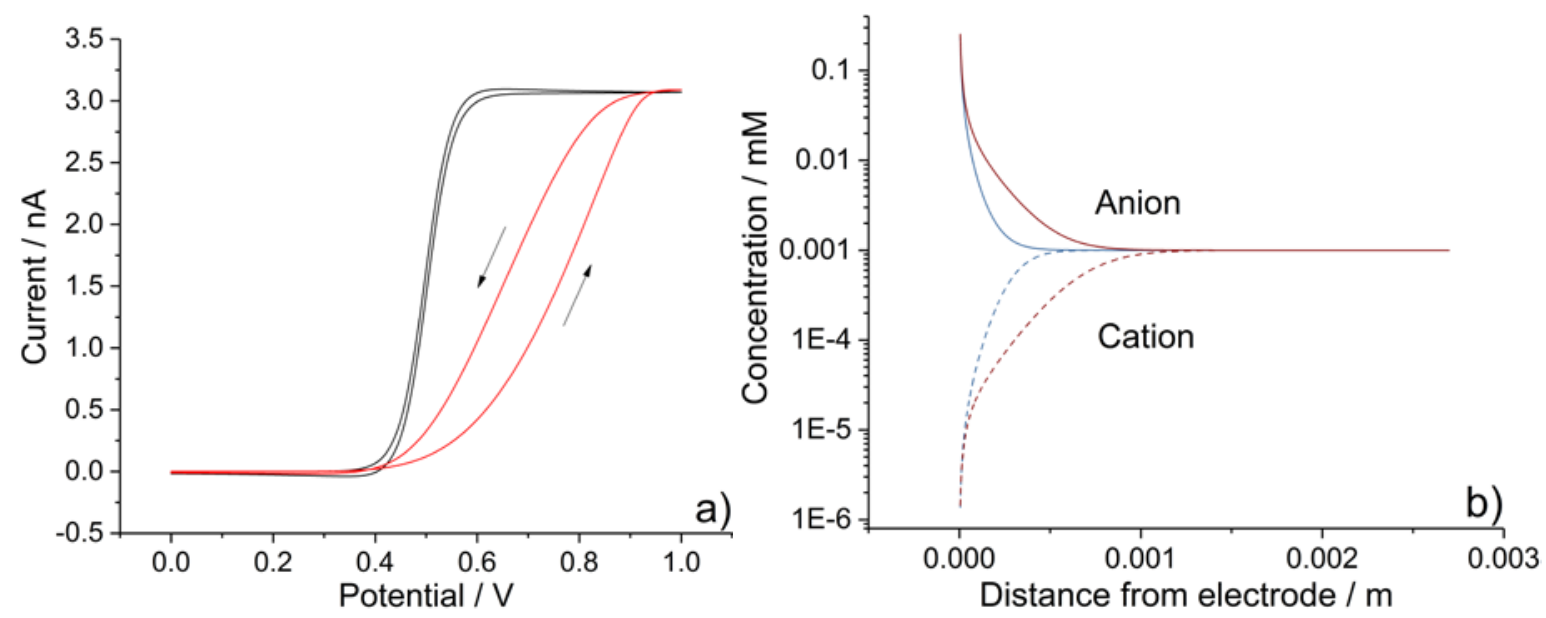

Figure 4: Reversible oxidation of $1 \mathrm{mM}$ analyte at a hemispherical electrode with a radius of $5 \mu \mathrm{m}, \mathrm{D}_{\mathrm{A}}=\mathrm{D}_{\text {sup }}=$ $1 \times 10^{-9} \mathrm{~m}^{2} \mathrm{~s}^{-1}, \mathrm{E}_{\mathrm{f}}=0.5 \mathrm{~V}$. a) voltammetric response in the presence of $100 \mathrm{mM}$ (black) and $1 \times 10^{-3} \mathrm{mM}(\mathrm{red})$ electrolyte. Arrows indicate the scan direction emphasising the voltammetric hysteresis. b) predicted concentration profiles at the half the steady-state current on the forward (blue) and reverse (dark red) voltammetric scan, the supporting electrolyte at a bulk concentration of $1 \times 10^{-3} \mathrm{mM}$.

Figure 4 a) depicts the simulated voltammetric response of a $5 \mu \mathrm{m}$ radius hemispherical electrode towards the oxidation of a $1 \mathrm{mM}$ solution of a redox active species in the presence of $100 \mathrm{mM}$ and $1 \times 10^{-3} \mathrm{mM}$ supporting electrolyte at a scan rate of $10 \mathrm{mV} \mathrm{s}^{-1}$, i.e. with a dimensionless scan rate of $9.7 \times 10^{-3}$. In the high support limit the voltammetric response exhibits the expected fully steady-state diffusional response (Figure 4 a) black line). Conversely, in the low support limit (Figure 4 a) red line), although at high overpotentials the voltammogram reaches the same diffusion limit current the voltammetric wave is distorted and shows significant hysteresis; the oxidation of the redox active species is easier on the backward scan as compared to the forward by approximately $110 \mathrm{mV}$. The origin of this discrepancy relates to the differing ionic concentration profiles on the forward and back scan of the voltammogram. Figure $4 \mathrm{~b}$ ) depicts the ionic concentration profiles for the supporting electrolyte in the vicinity of the electrode at a the half steady-state current on the forward and reverse scan, evidencing how even at this low scan rate the concentration profiles of the ionic species continue to evolve over the course of the voltammogram. To put this succinctly, attainment of the steady-state limit for a microelectrode depends not only upon the dimensionless scan rate but is also sensitive to the supporting electrolyte ratio. At low electrolyte concentrations rearrangement of the ionic species in the vicinity of the electrode takes a significant time period and becomes 
limited by the rate of mass-transport of the low concentration electrolyte to and from the electrode vicinity.

On the basis of Figure 3 for a hemispherical electrode system where all diffusion coefficients are equal the electrochemical cell is predicted to behave in accordance with Ohm's law (with less than a $20 \%$ variation in the cell resistance over the course of a scan) if $c_{\text {sup }} / c_{A} \geq 1$ and/or the dimensionless scan rate $\geq 390$. The timescale required to reach a steady-state response at a microelectrode in the absence of excess supporting electrolyte is longer than for a diffusion only system and depends upon the supporting electrolyte ratio and its diffusion coefficient.

\section{Conclusions}

Most electrochemical systems do not strictly behave in full accordance with Ohm's law and the cell resistance varies as a function of the electrochemical current. However, for macroelectrode systems and/or those with at least a support ratio of 1:1 the assumption that the cell resistance is a constant is likely tolerable and within experimental error. Systems in which the analyte and electrolyte diffusion coefficients differ significantly will need separate assessment. However, for microelectrode systems at low supporting electrolyte ratios the cell resistance can change significantly (by up to two orders of magnitude in the cases considered in this work) during the course of a scan. Consequently, commonly employed techniques for correcting Ohmic drop, such as the positive feedback technique as used in fast scan voltammetry, ${ }^{26}$ may not be applicable. The paper further demonstrates that additional complexity arises at low support ratios with the use of microelectrodes. The time required for the system to reach true steady-state behaviour in the absence of full support is significantly longer than that of a diffusion only system. 


\section{Acknowledgement}

This project is supported by the funding from the European Research Council under the European Union's Seventh Framework Programme (FP/2007-2013)/ERC Grant Agreement no. [320403].

\section{References}

1. Newman, J.; Thomas-Alyea, K. E., Electrochemical Systems; John Wiley \& Sons, 2012.

2. Newman, J., Resistance for Flow of Current to a Disk. 1965.

3. Holm, R.; Holm, E., Electric Contacts; Theory and Application, 4th completely rewritten ed.; Springer-Verlag: Berlin ; New York, 1967.

4. Maxwell, J. C., A Treatise on Electricity and Magnetism, 3rd ed.; Clarendon Press: Oxford, 1904.

5. Strutt, J. W., V. On the Theory of Resonance. Philosophical Transactions of the Royal Society of London 1871, 161, 77-118.

6. Aoki, K. J.; Chen, J., Insight of Electrolyte-Free Voltammetry at Microelectrodes. Current Opinion in Electrochemistry 2018.

7. Ciszkowska, M.; Stojek, Z., Voltammetry in Solutions of Low lonic Strength. Electrochemical and Analytical Aspects. Journal of Electroanalytical Chemistry 1999, 466, 129-143.

8. Oldham, K. B., Theory of Microelectrode Voltammetry with Little Electrolyte. Journal of Electroanalytical Chemistry 1988, 250, 1-21.

9. Amatore, C.; Deakin, M. R.; Wightman, R. M., Electrochemical Kinetics at Microelectrodes: Part Iv. Electrochemistry in Media of Low lonic Strength. Journal of electroanalytical chemistry and interfacial electrochemistry 1987, 225, 49-63.

10. Bond, A. M.; Fleischmann, M.; Robinson, J., Electrochemistry in Organic Solvents without Supporting Electrolyte Using Platinum Microelectrodes. Journal of Electroanalytical Chemistry and Interfacial Electrochemistry 1984, 168, 299-312.

11. Belding, S. R.; Laborda, E.; Compton, R. G., Steady-State Voltammetry at a Microdisc Electrode in the Absence of Excess Supporting Electrolyte for Reversible, Quasi-Reversible and Irreversible Electrode Kinetics. Physical Chemistry Chemical Physics 2012, 14, 14635-14649.

12. Li, X.; Batchelor - McAuley, C.; Laborda, E.; Compton, R. G., Aqueous Voltammetry in the near Absence of Electrolyte. Chemistry-A European Journal 2017, 23, 15222-15226. 
13. Jiao, X.; Batchelor-McAuley, C.; Lin, C.; Kätelhön, E.; Tanner, E. E. L.; Young, N. P.; Compton, R. G., The Role of Nanomorphology and Interfacial Structure of Platinum Nanoparticles in Catalysing the Hydrogen Oxidation Reaction. ACS Catalysis 2018.

14. Krause, K. J.; Brings, F.; Schnitker, J.; Kätelhön, E.; Rinklin, P.; Mayer, D.; Compton, R. G.; Lemay, S. G.; Offenhäusser, A.; Wolfrum, B., The Influence of Supporting lons on the Electrochemical Detection of Individual Silver Nanoparticles: Understanding the Shape and Frequency of Current Transients in Nano - Impacts. Chemistry - A European Journal 2017, 23, 4638-4643.

15. Ngamchuea, K.; Clark, R. O. D.; Sokolov, S. V.; Young, N. P.; Batchelor - McAuley, C.; Compton, R. G., Single Oxidative Collision Events of Silver Nanoparticles: Understanding the Rate - Determining Chemistry. Chemistry - A European Journal 2017, 23, 16085-16096.

16. Compton, R. G.; Laborda, E.; Ward, K. R., Understanding Voltammetry: Simulation of Electrode Processes; World Scientific Publishing Company, 2013.

17. Dickinson, E. J. F.; Limon-Petersen, J. G.; Rees, N. V.; Compton, R. G., How Much Supporting Electrolyte Is Required to Make a Cyclic Voltammetry Experiment Quantitatively "Diffusional"? A Theoretical and Experimental Investigation. The Journal of Physical Chemistry C 2009, 113, 11157-11171.

18. Dickinson, E. J. F.; Compton, R. G., The Zero-Field Approximation for Weakly Supported Voltammetry: A Critical Evaluation. Chemical Physics Letters 2010, 497, 178-183.

19. Imbeaux, J. C.; Savéant, J. M., Linear Sweep Voltammetry. Effect of Uncompensated Cell Resistance and Double Layer Charging on Polarization Curves. Journal of Electroanalytical Chemistry and Interfacial Electrochemistry 1970, 28, 325-338.

20. van der Vliet, D.; Strmcnik, D. S.; Wang, C.; Stamenkovic, V. R.; Markovic, N. M.; Koper, M. T. M., On the Importance of Correcting for the Uncompensated Ohmic Resistance in Model Experiments of the Oxygen Reduction Reaction. Journal of Electroanalytical Chemistry 2010, 647, 29-34.

21. Here we are not referreing to the potential drop across the double layer but the drop induced by the occurence of the electrochemical reaction. This relates to the so-called 'primary' current distribution which is obtained from solving the Laplace equation and the relevant length scale is that of the electrode.

22. Oldham, K. B., All Steady-State Microelectrodes Have the Same "Ir Drop". Journal of Electroanalytical Chemistry 1987, 237, 303-307.

23. The electrolyte is not created or destroyed at the electrode surfaces (working or counter) hence the flux of the species into and out of the system is zero. From conservation of mass this necessarilly implies that at steady-state there is no overall flux of electrolyte through the cell and hence the electrolyte does not carry any of the ionic current.

24. Note equation 23 differs from equation 34 presented by Oldham in reference $X$, this later expression for the half wave potential further accounts for the alteration in the voltammetric waves position as a function of the mass-transport properties. 
25. Hyk, W.; Palys, M.; Stojek, Z., Migrational Chronoamperometry of Uncharged Substrates. Influence of Electron Transfer Rate. Journal of Electroanalytical Chemistry 1996, 415, 13-22.

26. Garreau, D.; Hapiot, P.; Savéant, J. M., Fast Cyclic Voltammetry at Ultramicroelectrodes: Current Measurement and Ohmic Drop Positive Feedback Compensation by Means of Current Feedback Operational Amplifiers. Journal of Electroanalytical Chemistry and Interfacial Electrochemistry 1990, 281, 73-83. 